\title{
Percepção dos enfermeiros na assistência em cuidados paliativos
}

\author{
Perception of nurses in palliative care assistance \\ Percepción de las enfermeiras en la asistencia de cuidados paliativos
}

Recebido: 11/12/2020 | Revisado: 17/12/2020 | Aceito: 30/12/2020 | Publicado: 03/01/2021

\author{
Daniele Beal Arnauts \\ ORCID: https://orcid.org/0000-0003-0930-4724 \\ Universidade Paranaense, Brasil \\ E-mail: daniarnauts@gmail.com \\ Jolana Cristina Cavalheiri \\ ORCID: https://orcid.org/0000-0002-9549-8985 \\ Universidade Paranaense, Brasil \\ E-mail: Jolana_cc@hotmail.com
}

\begin{abstract}
Resumo
O objetivo do estudo foi identificar a percepção dos enfermeiros acerca da assistência ao paciente em cuidado paliativo. Fez-se uma pesquisa exploratória, descritiva, de campo, com caráter qualitativo. Participaram da pesquisa 15 enfermeiros de um hospital privado do Paraná. Os dados foram coletados por meio de entrevista semiestruturada, coletando dados sociodemográficos e sobre a percepção dos profissionais em relação aos cuidados paliativos. Os resultados foram analisados pelo Método de Bardin. Observou-se que os profissionais referem o cuidado paliativo como aquele que oferece conforto e alívio da dor, possui participação da família e é respaldado por uma equipe multidisciplinar, para a qual há necessidade de protocolos e treinamentos. Os enfermeiros possuem uma visão limitada em relação à assistência ao cuidado paliativo, o que dificulta a prestação de um cuidado qualificado.
\end{abstract}

Palavras-chave: Cuidados paliativos; Enfermagem; Cuidados paliativos na terminalidade da vida.

\begin{abstract}
The objective of the study was to identify the nurses' perception of patient care in palliative care. This is an exploratory, descriptive, field research, with a qualitative character, 15 nurses from a private hospital in Paraná participated in the research. Data were collected through semi-structured interviews, containing sociodemographic variables and the professionals' perception of palliative care. The results were analyzed using the Bardin method. It was observed that professionals refer to palliative care as one that offers comfort and pain relief, has family participation, is supported by a multidisciplinary team, in which the need for protocols and trained staff is observed. Nurses have a limited view regarding assistance to palliative care, which makes it difficult to provide qualified care.
\end{abstract}

Keywords: Palliative care; Nursing; Hospice care.

\section{Resumen}

El objetivo del estudio fue identificar la percepción de las enfermeras sobre el cuidado del paciente en cuidados paliativos. Se trata de una investigación de campo, exploratoria, descriptiva, de carácter cualitativo, participaron 15 enfermeras de un hospital privado de Paraná. Los datos fueron recolectados a través de entrevistas semiestructuradas, conteniendo variables sociodemográficas y percepción de los profesionales sobre los cuidados paliativos. Los resultados se analizaron mediante el método de Bardin. Se observó que los profesionales se refieren a los cuidados paliativos como aquellos que ofrecen comodidad y alivio del dolor, tiene participación familiar, es apoyado por un equipo multidisciplinario, en el cual se observa la necesidad de protocolos y personal capacitado. Las enfermeras tienen una visión limitada sobre la asistencia a los cuidados paliativos, lo que dificulta la prestación de una atención calificada.

Palabras clave: Cuidados paliativos; Enfermería; Cuidados paliativos al final de la vida.

\section{Introdução}

A Organização Mundial da Saúde (OMS) define como idoso o indivíduo cuja idade ultrapassa os 60 anos. O Brasil teve seu perfil demográfico alterado a partir da década de 70, quando famílias rurais, numerosas e com taxas altíssimas de mortalidade passaram a ser urbanas e com menor quantidade de filhos, promovendo aumento da perspectiva de vida e crescimento acentuado da população idosa (Miranda, Mendes \& Silva, 2016).

Embora envelhecer não esteja associado a adoecer, o aumento da expectativa de vida está relacionado ao surgimento 
de doenças crônicas não transmissíveis (DCNT), responsáveis por 70\% dos óbitos mundiais. Dentre estas, destacam-se as doenças cardiovasculares, respiratórias, cânceres e diabetes. São as principais patologias que, além da morte, causam a perda da qualidade de vida, impactos negativos na economia e nas relações familiares e sociais, além de estarem associadas à necessidade de assistência prolongada (Malta et al., 2020). Sendo assim, juntamente com a senilidade, a queda dos sistemas orgânicos também facilita o desenvolvimento de doenças que ameaçam a vida e, consequentemente, tem-se a necessidade dos cuidados paliativos (Queiroga, Menezes, Lima \& Andrade, 2020).

Cuidados paliativos são entendidos como serviços prestados por uma equipe multidisciplinar com objetivo de melhorar a qualidade de vida do paciente e da família, diante do diagnóstico de uma doença que ameace a vida (OMS, 2019). O alívio do sofrimento, o conforto, o tratamento da dor e dos inúmeros sintomas associados - sejam físicos, espirituais, sociais ou psicológicos - tornam-se os objetos do tratamento (Santos et al., 2020).

A prática de cuidado paliativo pode ser desenvolvida em âmbito ambulatorial, hospitalar ou domiciliar, e tem como principal objetivo fornecer um cuidado profissional e qualificado, reduzindo o número de internações e de manipulações desnecessárias ao paciente (Queiroga, Menezes, Lima \& Andrade, 2020).

Entretanto, a influência do modelo biológico leva os pacientes em cuidados paliativos a findar suas vidas em leitos da Unidade de Terapia Intensiva (UTI) e outros serviços hospitalares, o que exige da equipe multidisciplinar uma busca incessante na melhoria da qualidade de vida dessas pessoas. Todos os membros da equipe pontuam um único objetivo, o alívio da dor e dos sintomas associados, no entanto, cada categoria possui uma forma de otimizar esses cuidados (Pires et al., 2020).

Diante disso, a participação da enfermagem na assistência aos cuidados paliativos requer do enfermeiro uma sistematização eficaz do cuidado, bem como o planejamento e a implantação de ações que visam a promover a autonomia do paciente junto com a família, sobre sua própria doença (Meireles et al., 2020). Além disso, o enfermeiro que atua nessa área deve analisar as necessidades e avaliar como o cliente e a família estão vivendo perante a situação, sendo esses fatores primordiais na construção de um cuidado com qualidade (Santos et al., 2020).

Diante da necessidade de cuidados paliativos crescentes na população e a execução de um cuidado qualificado por parte do enfermeiro, sendo capaz de avaliar o indivíduo diariamente e manter ou incluir uma nova terapêutica (Coelho \& Yankaskas, 2016), este estudo questionou "qual a percepção e o conhecimento da enfermagem em cuidados paliativos no ambiente hospitalar?"

Neste contexto, o objetivo desta pesquisa foi identificar a perspectiva dos enfermeiros sobre cuidados paliativos em ambiente hospitalar.

\section{Métodos}

Trata-se de um estudo exploratório, descritivo, de campo, com caráter qualitativo, por meio de entrevistas realizadas com enfermeiros atuantes no serviço hospitalar.

As pesquisas exploratórias e descritivas tem por objetivo proporcionar uma maior familiaridade com o tema estudado e descrever os fatores que determinam a ocorrência dos fenômenos. Já o estudo de campo visa aprofundar as questões propostas no grupo pesquisado, ressaltando a interação entre os componentes, enquanto o estudo qualitativo é aquele no qual os dados são coletados de forma descritiva, preocupando-se em dar significado aos questionamentos realizados, sendo o pesquisador, o principal instrumento da coleta de dados (Pereira, Shitsuka, Parreira \& Shitsuka, 2018)

O município de escolha para realização da pesquisa foi Francisco Beltrão, localizado no Sudoeste do Paraná, que segundo o Instituto Brasileiro de Geografia e Estatística (IBGE, 2019) possui 91.093 habitantes e uma área territorial de $735,111 \mathrm{~km}^{2}$ (2018), abrangendo 45 estabelecimentos de saúde (2009). A instituição hospitalar escolhida foi um hospital 
privado que presta atendimento à Oitava Regional de Saúde do Paraná, cuja estrutura conta com diversas unidades, como UTI, emergência e serviços clínicos.

Constituíram a população deste estudo todos os enfermeiros atuantes na UTI e nas unidades clínicas, somando um total de 15 enfermeiros, sendo a amostragem por conveniência. Os critérios de inclusão consideraram os enfermeiros atuantes nas unidades supracitadas há mais de 30 dias, que atuavam somente na assistência e aceitaram participar da pesquisa, mediante assinatura do Termo de Consentimento Livre e Esclarecido (TCLE). Foram exclusos os enfermeiros com atestado médico, em licença à maternidade, os ausentes no momento da coleta de dados e os que não aceitaram participar da pesquisa.

A coleta de dados foi realizada entre os meses de agosto e setembro de 2020, por meio de entrevista. Para os enfermeiros da UTI ela foi realizada no ambiente de trabalho, durante o intervalo dos profissionais, em uma sala reservada, respeitando a privacidade dos participantes. Para os demais profissionais foi programado um cronograma de coleta, sendo realizado conforme a sua disponibilidade, após o término do plantão, respeitando as normas de proteção e distanciamento social, tendo em vista a pandemia enfrentada no país.

O instrumento para a coleta de dados foi uma entrevista semiestruturada composta por duas partes. A primeira parte tinha por objetivo avaliar as variáveis sociodemográficas, tais como idade, sexo, renda familiar, estado civil, número de vínculos empregatícios, turno de trabalho, setor de trabalho, tempo de experiência, tempo de serviço, se possuía especialização e treinamento diante do assunto. A segunda parte era constituída de perguntas para compreender a percepção dos enfermeiros acerca dos cuidados paliativos, sendo elas: Qual seu entendimento acerca da assistência ao paciente em cuidado paliativo? Você já vivenciou essa assistência nessa unidade? Se sim, como foi? Como foi sua relação com a família? Você acha que pode melhor assistir essa clientela? De que modo? Do que você precisa? Que ações/cuidados você considera próprias do cuidado paliativo?

As entrevistas foram gravadas com dispositivo MP3 e posteriormente transcritas na íntegra. Após a transcrição elas foram submetidas à categorização pelo Método de Bardin e após a análise todo material utilizado foi destruído. Para descrição das falas, utilizou-se o termo "Enfermeiro" com a numeração da entrevista, em ordem crescente.

O trabalho foi aprovado pelo Comitê de Ética em Pesquisa com Seres Humanos da Universidade Paranaense, sob Protocolo n. 4.055.496, em maio de 2020, e seguiu as recomendações éticas em pesquisa com seres humanos de acordo com a Resolução n 466/2012 do Conselho Nacional de Saúde.

\section{Resultados}

Participaram da pesquisa 15 enfermeiros, sendo 13 mulheres e 2 homens, com idade variando de 25 a 45 anos, em sua maioria solteiros, com renda familiar de dois a três salários mínimos. Prevaleceu a duplicidade de vínculo empregatício, com turno de trabalho variado, tempo de serviço de um a 15 anos e na profissão de um a 20 anos.

Quanto à especialização, a maioria dos profissionais relatou tê-la, com enfoque em Urgência e Emergência e em UTI. Em relação ao conhecimento e treinamento em Cuidados Paliativos, 13 profissionais referiram não o possuir.

Quando questionados sobre qual a percepção e conhecimento acerca dos cuidados paliativos no ambiente hospitalar, desprenderam-se quatro categorias: "Alívio e conforto da dor", "Participação da família", "Equipe multidisciplinar" e "Protocolos e equipe treinada".

\section{Alívio e conforto da dor}

A primeira categoria "Alívio e conforto da dor" surgiu do questionamento sobre o entendimento do profissional quanto à assistência em cuidado paliativo. Sobre isso se observou que o conforto e alívio da dor foram as principais medidas instituídas pelos profissionais, conforme os discursos abaixo: 
"[...] meu entendimento a assistência em cuidado paliativo tem que tentar dar o máximo possível de conforto ao paciente, pra ele não sentir dor, é, promover assim que ele se sinta o mais confortável possivel [...]”. (Enfermeiro 7). "[...] mas aquele a cuidado paliativo deve ficar bem, fique sem dor, né, uma atenção mais carinhosa, um alívio da dor, um alívio da parte psicológica". (Enfermeiro 11).

“[...] o paciente em cuidado paliativo não quer dizer que ele está lá pra esperar a hora dele, entende? [...] só que esse cuidado tem que ser um cuidado bem prestado e com excelência, pra pelo menos amenizar a dor dele e sofrimento [...]”. (Enfermeiro 10).

"Proporcionar medidas de conforto, minimizando sofrimento fisico e psicológico, oferecendo qualidade vida do paciente." (Enfermeiro 15).

\section{Participação da família no cuidado paliativo}

A segunda categoria foi intitulada "Participação da família no cuidado paliativo", na qual a maioria dos profissionais reiterou a importância dos familiares neste processo, conforme as falas abaixo:

'[...] companhia da família, porque o paciente se sente mais confortável, mais seguro quando a família está por perto. Principalmente quando são pessoas que ele tem mais contato, acho que isso ajuda na terapêutica [...]”. (Enfermeiro 15).

"[...] acho que a gente pode, é mante os familiares sempre perto, e fazer coisas que o paciente talvez gostava de ter perto [...]”. (Enfermeiro 13).

"[...] também um trabalho junto às familias, né? Elas ter o tempo da visita, estar ao lado dos seus familiares ali nesse momento pra que ambos se confortem". (Enfermeiro 6).

"[...] eu acho que tem que ficar num quarto assistido com a família junto, com medidas de conforto, pra dor, e não deixar isolado dentro da UTI pra vir falecer. Ter mais humanização com a família [...]”. (Enfermeiro 2).

\section{Equipe multidisciplinar}

A terceira categoria foi denominada como "Equipe multidisciplinar", visto que os profissionais relataram ser de extrema importância sua participação e engajamento na assistência aos pacientes paliativos.

"[...] a gente precisa de equipes multidisciplinares, tem que ter apoio psicológico, tanto pra família quanto pro paciente, tem que ter a assistente social, assim como o atendimento do médico e da enfermagem [...]". (Enfermeiro $15)$.

"[...] é dando assistência a ele e a família, isso agregar psicólogo, fisioterapeuta, equipe de enfermagem, médicos, nutricionistas, enfim toda equipe que é necessário para o paciente se sentir confortável”. (Enfermeiro 6).

"[...] Além de uma equipe multidisciplinar né, por que nesses cuidados dentro do hospital toda a equipe é importante, aí entra os psicólogos né, os fisios, a nutricionista". (Enfermeiro 8). 


\section{Protocolos e equipe treinada}

A quarta categoria foi nomeada como "Protocolos e equipe treinada", tendo em vista que os profissionais pontuaram os protocolos e o treinamento como condutas mais necessárias a serem tomadas para melhorar a assistência prestada ao paciente paliativo, conforme os discursos abaixo:

“[...] primeiro treinar a equipe, é... ter uma equipe completa, preparada, com tempo hábil pra ficar à beira do leito, trabalhando com esses pacientes [...]”. (Enfermeiro 6).

“[...] acho assim, que a gente tinha que ter cursos, né? Ter capacitação em relação a cuidados paliativos[...] Todo mundo falando a mesma língua, tudo certinho. Acho que é isso, acho que falta uma capacitação”. (Enfermeiro 11).

"[...] desde que toda equipe fale a mesma língua, toda equipe seja treinada pra isso, né? [...] mas a equipe, principalmente, que todo mundo fale a mesma língua, aí pode ser bem assistido. Eu acho que é treinamento, entendimento disso[...]". (Enfermeiro 3).

\section{Discussão}

Os cuidados paliativos vêm se tornando uma realidade cada vez mais frequente nos serviços de saúde. Dessa forma, este estudo proporcionou uma reflexão por parte dos profissionais, que realçaram a importância de aprofundamento em relação ao conhecimento em cuidados paliativos. Como limitação do estudo destaca-se a escolha de uma única instituição hospitalar do município - o que impede a generalização dos dados - bem como o foco no atendimento clínico e cirúrgico por parte da assistência ofertada pelo serviço.

Quanto ao perfil dos profissionais, prevaleceram mulheres, com idade de 25 a 45 anos, solteiras, com renda familiar de dois a três salários mínimos, com mais de um emprego e turno de trabalho variado, com tempo de experiência diversificado na instituição e na enfermagem. Esses dados vão ao encontro dos obtidos em estudo desenvolvido em hospital filantrópico de Minas Gerais, no qual prevaleceu o sexo feminino, com idade de 26 a 46 anos, que trabalhavam durante o dia e possuíam tempo médio de 5,6 anos trabalhando na instituição (Barbosa et al., 2020).

Também se observou que a maioria dos profissionais possuía especialização em cuidados intensivos, o que discorda de um estudo realizado em um hospital que prestava atendimento a pacientes terminais em Teresina - PI, no qual a maioria dos enfermeiros não possuía especialização (Silva et al., 2020).

Da mesma forma, 13 profissionais aludiram não ter treinamento específico em cuidados paliativos, o que vai ao encontro de outra pesquisa na qual os participantes também referiram não ter conhecimento suficiente em cuidados paliativos e sentirem-se inseguros durante sua prática (Amorim et al., 2020). O hospital onde foi realizada a presente pesquisa oferta atendimento clínico e cirúrgico, o que possivelmente faz com que os profissionais busquem especializações em áreas relacionadas e evitem ou não se interessem em aprofundar seu conhecimento em cuidados paliativos, mesmo que, eventualmente, tenham contato com esses pacientes.

A primeira categoria do estudo diz respeito à "percepção dos profissionais sobre os cuidados paliativos". Nesta categoria eles referiram-se ao conforto e ao alívio da dor, e ainda que esses sejam princípios fundamentais desta ótica de cuidado, demonstraram uma visão reduzida e fragmentada da assistência de enfermagem na sua aplicação.

O cuidado paliativo foi desenvolvido com o propósito de oferecer assistência e melhoria na qualidade de vida do paciente, sendo indicado para crianças ou adultos cuja doença ameace a vida, e tem como objetivo a valorização do viver, bem como o enfrentamento da morte como um processo natural, considerando o indivíduo em seus aspectos biopsicossociais (Silva et al., 2020). Além disso, esse tipo de cuidado se apresenta através de práticas que proporcionam a minimização da dor, além 
de outros sintomas associados - que podem ser físicos, espirituais, sociais ou psicológicos - e é aplicado desde o diagnóstico até o momento do luto (Paiva et al., 2019).

A prática de cuidado paliativo no Brasil ainda se mostra limitada, baseando-se na modalidade de assistência à terminalidade da vida, pois nesse estágio da doença entende-se que a patologia é irreversível. Logo, a mudança desse pensamento é um processo que depende dos profissionais atuantes na área e de uma formação holística na graduação (Silveira et al., 2016).

Sendo assim, percebeu-se neste estudo que o conforto e alívio da dor foram as principais medidas aludidas pelos enfermeiros. Ressalta-se que a dor é o quinto dos sinais vitais, sendo descrita como um processo fisiológico associado a uma experiência impertinente, adjunta a lesões reais ou potenciais e, da mesma forma, influente na qualidade de vida do paciente (Corgozinho, Barbosa, Araújo \& Araújo, 2020), consistindo em uma das maiores causas de sofrimento humano, provocando reflexões sobre seu estado físico e psicossocial (Sousa \& Nunes, 2019).

Em 1980, a OMS posicionou-se frente aos pacientes em cuidados paliativos, elencando a dor como uma emergência médica mundial (Almeida, Gama, Espejo \& Pedroso, 2018). Nessa perspectiva, promover o alívio da dor e proporcionar conforto para os pacientes em cuidados paliativos é uma intervenção da prática de enfermagem, a qual exige não só conhecimento científico, mas humanização e ética, além da habilidade técnica (Nascimento, 2017).

Sabendo que a equipe de enfermagem é quem passa a maior parte do tempo com o paciente, o enfermeiro deve estar capacitado para avaliar e identificar as causas e possíveis condutas perante a dor do cliente (Reis \& Reis 2017). Dentro do manejo da dor existem duas possibilidades: as intervenções farmacológicas e não farmacológicas. A primeira consiste na administração de medicamentos cuja única finalidade é o alívio e controle da dor e o conforto; faz-se uso de analgésicos, sendo os mais utilizados os opioides, os não opioides e os sedativos (Andrade et al., 2018). Já a segunda - mesmo que ainda encontre muitas divergências por parte dos próprios profissionais - é muito utilizada em associação à primeira, sendo caracterizada por procedimentos como: mudança de decúbito, menor manuseio do paciente, musicoterapia, criação de vínculo entre paciente, família e profissional (Silva, Braga, Sena, Duarte \& Sena, 2019), oferta de dieta e de líquidos, hidratação da pele para evitar lesão por pressão, segurança, tranquilidade, confiança para a família, proporcionar que o paciente fique com seus entes queridos em um ambiente agradável e seguro, ouvir o paciente, deixando-o desabafar sobre seus medos e inseguranças (Santos et al., 2020).

Observou-se neste estudo que um dos profissionais citou a necessidade de cuidar a dor do paciente, incluindo da parte psicológica, o que demonstra que a dor em cuidados paliativos envolve muito mais do que o aspecto físico, pois além de ser muito relatada pelos pacientes, ela compreende os aspectos emocionais, sociais, espirituais e psicológicos, e a esse conjunto de elementos denomina-se dor total. Contudo, o alívio da dor requer atenção especial de toda a equipe que presta assistência para o paciente (Nascimento, Campos, Vieira \& Barbosa, 2020).

Dessa forma, os profissionais devem ser capazes de identificá-la e agir sobre ela, deparando-se não só com a dor do paciente, mas também com a família. A equipe deve articular o seu conhecimento e determinar um plano terapêutico, propondo ações que visam ao alívio da dor em todo o seu aspecto, garantindo a qualidade de vida de ambos (Leite et al., 2020).

Ressalta-se, ainda, que os cuidados paliativos não podem ser confundidos com a eutanásia, visto que seu significado é o cuidado de qualidade até o fim da vida, excluindo a ideia precipitada de que não há mais nada a ser feito (Lóos, Dias, Cabral \& Souza, 2019), fato também mencionado pelos profissionais.

A segunda categoria foi intitulada "a participação da família no cuidado paliativo", consistindo em um dos princípios básicos deste cuidado, no qual o paciente pode desfrutar de seus dias com dignidade, se possível em casa e ao lado de pessoas que o amam (Brandão \& Góis, 2020). 
Diante disso, a adesão da família no cuidado paliativo se torna um suporte fundamental para que o paciente consiga enfrentar a doença e as próximas decisões que serão tomadas acerca de seu tratamento, pois, nessa fase, o paciente passa por uma mistura de sentimentos, como aceitação, negação, raiva e frustrações (Martins et al., 2020), por isso é muito importante possuir o familiar ao lado para prestar-lhe apoio.

Também se percebeu no discurso dos profissionais uma preocupação quanto a não deixar o paciente isolado ou falecer sozinho na UTI. Nesse contexto a Organização Mundial da Saúde, desde a década de 90, enfoca a internação domiciliar como prioritária para pacientes em cuidados paliativos, visto que essa alternativa, além de diminuir o custo com leitos hospitalares, promove a convivência familiar e o afeto, trazendo melhorias na qualidade de vida do paciente (Simão \& Mioto, 2016).

Sabendo que os cuidados paliativos não dizem respeito somente ao paciente, mas que englobam também os familiares, a comunicação entre equipe, família e paciente é um componente de suma importância, visto que traz efeitos positivos para o enfrentamento desse processo (Santos, Silva \& Ribeiro, 2020). Tendo em vista que o processo de internamento expõe o paciente à vulnerabilidade, sobretudo nas pessoas mais idosas, a presença da família é fundamental, pois remete à proteção. Os laços familiares que se desenvolveram ao longo dos anos auxiliam o paciente a aceitar o processo pelo qual está passando, o que implica diretamente na formação de vínculos com a equipe que presta esses cuidados (Queiroz et al., 2018).

Porém, a família também precisa de suporte. Nesse sentido a atuação da equipe multiprofissional permite a construção de vínculo para que os familiares sintam-se à vontade para falarem de seus sentimentos diante da vivência e enfrentamento da doença, da morte e do luto, bem como para receberem informações sobre os cuidados com o paciente, a fim de que tirem de suas costas o peso do cansaço que a demanda desse cuidado traz (Monteiro et al., 2020).

Os discursos dos participantes também corroboraram com estudo realizado em uma unidade de internação pediátrica, o qual ressaltou a participação da família, que pode ficar esquecida devido à patologia do paciente, porém, é uma fonte de cuidado que precisa de apoio e de alguém que os incentive (Carvalho, Vieira, Tacla, Misael \& Barros, 2020). Esse fato foi ressaltado pelo enfermeiro 6, que destacou a necessidade de cuidado também ao familiar, já que neste processo pode sofrer de luto antecipado ou mesmo adoecer junto com o ente querido.

Sabendo que nesse momento os familiares estão igualmente vulneráveis, é necessária uma atenção ampla, inserindoos no contexto do cuidado. Nesse sentido, a relação do enfermeiro com a família deve ocorrer de forma transparente e humanizada, focando nas formas física, social, emocional, ética e espiritual (Santos, Lira \& Costa, 2018).

Quanto à terceira categoria, observou-se que os enfermeiros ressaltaram a necessidade de uma equipe multiprofissional para atuar em cuidados paliativos, o que concorda com os achados de um estudo bibliográfico que ressaltou a importância de uma equipe formada por diversos profissionais na assistência para esse tipo de cuidado, sendo necessário que a equipe seja ativa e olhe para o paciente como um todo, considerando seus aspectos sociais, morais e psicológicos (Pulga et al., 2019). Além disso, a atuação de diversos profissionais pode ser implementada nos diferentes estágios dos cuidados paliativos, desde o diagnóstico, passando pelo cuidado com a família, até os cuidados no fim da vida (Paiva et al, 2020).

Tendo em vista a essência da atuação de uma equipe ampla nesse tipo de atendimento, os profissionais que englobam essa equipe são basicamente médicos, enfermeiros, técnicos de enfermagem, farmacêuticos, psicólogos, assistentes sociais, fonoaudiólogos, dentistas e fisioterapeutas, e cada um deles atua em sua área específica, porém, seguindo sempre a mesma linha de raciocínio e a mesma lógica de cuidado (Borba, 2020). Além disso, ainda podem estar presentes acompanhantes religiosos e terapeutas diversos.

Dado que um dos princípios do cuidado paliativo é o alívio da dor e do sofrimento em todas as suas proporções, é necessário que a equipe trabalhe em sintonia e que verbalize com o paciente e a família, de forma clara e objetiva, para um melhor enfrentamento (Pupim, 2018). Ademais, os profissionais precisam estar atentos aos familiares, uma vez que a notícia 
do adoecimento de um ente querido pode levar ao adoecimento também da família, que fica continuadamente sofrendo com a mudança de rotina e com as difíceis tomadas de decisão (Lima, 2019).

Sendo assim, a enfermagem destaca-se nesse processo, pois o enfermeiro e a equipe são os profissionais que passam a maior parte do tempo com o paciente durante a internação, o que engloba uma prática de cuidados singular, com assistência planejada e qualificada, e os torna protagonistas para o paciente e a família, proporcionando alívio dos sintomas e qualidade de vida aos envolvidos (Silva et al., 2020; Silveira, da, Costa, da, Lohmann \& Lavall, 2020).

O enfermeiro é ampla e cientificamente qualificado e técnico para prestar esse tipo de atendimento, e sua atividade paliativa é essencial, levando em conta muitas ferramentas, dentre elas a comunicação (Ribeiro, 2017). Certamente, uma boa relação entre a tríade paciente, família e enfermeiro é uma forma terapêutica e eficaz na abordagem ao cuidado, visto que este profissional está à frente na hora de notificar os familiares, seja com boas, ou más notícias, demonstrando destreza e sensibilidade (Gomes, 2019).

O planejamento da assistência é complexo e trabalhoso e reúne questões éticas, entre definir se existe a reversibilidade do caso ou não, e quais decisões serão tomadas posteriormente (Santos et al., 2017).

A quarta categoria foi nomeada como "Protocolos e equipe treinada" e percebeu-se pelos depoimentos dos participantes a importância de uma equipe com conhecimento para atuação em cuidados paliativos. Esse fato corrobora com o estudo realizado em uma UTI em Santarém - PA, que apontou a importância do aperfeiçoamento da equipe em relação a suas habilidades, sejam técnicas ou científicas, bem como a necessidade de planejamento da assistência prestada (Aquino; Góes \& Malcher, 2018).

No Brasil já se ouvia falar em cuidados paliativos desde os anos 80, porém de forma isolada. Somente em 2017 a atuação em cuidados paliativos foi reconhecida pela Associação Médica Brasileira, e em 2018 o Ministério da Saúde publicou uma resolução que normatiza os cuidados paliativos como parte dos cuidados integrados no SUS (Lourençato, 2020).

No entanto, apesar de os profissionais ressaltarem a importância de protocolos, os cuidados paliativos incluem especificamente alguns princípios, os quais ressaltam que: a morte deve ser vista como processo natural e que, nesse sentido, a qualidade de vida do paciente é mais importante; os cuidados paliativos não antecipam a morte, muito menos prolongam o processo de morrer; a família deve ser inclusa nesse cuidado; controlar os sintomas através da avaliação rotineira é fundamental; paciente e família têm direito a informações e opções de tratamento, sendo que as decisões devem ser compartilhadas e de maneira ética; cuidados paliativos são desenvolvidos necessariamente por equipe multidisciplinar, a assistência é global e não se encerra com a morte do paciente, mas se estende com o luto da família (Gomes \& Othero, 2016).

Dessa forma, apesar de os participantes sugerirem a necessidade de protocolos assistenciais, entendendo-se que cada paciente e família são únicos, é essencial que o cuidado ofertado pela equipe volte-se para alguns princípios norteadores que são: iniciar o mais rápido possível o acompanhamento do paciente, para compreender qual é o melhor manejo; reafirmar a vida e a importância de sua qualidade; promover o alívio impecável de todos os sintomas que geram desconforto; oferecer o melhor suporte para o paciente e para a família, para que possa viver ativamente e com autonomia até a sua morte; incluir os familiares e os entes queridos nesse processo, dando suporte e apoio durante a fase do adoecimento até o luto (Manual de Cuidados Paliativos, 2020).

Sabendo que a expectativa de vida está aumentando e com ela o envelhecimento populacional e as altas taxas de doenças crônicas não transmissíveis, tem-se a necessidade do cuidado paliativo. Estima-se que no Brasil entre 456 e 537 para cada 100.000 habitantes necessitam desse tipo de cuidado, no entanto, muitas vezes essa assistência é fornecida dentro das unidades hospitalares devido ao despreparo da atenção primária (Queiroga et al., 2020).

A assistência ao paciente paliativo em domicílio enfrenta algumas divergências como a falta de tempo dos profissionais, a sua falta durante o fim de semana e os feriados, a falta de informação do paciente e da família. Além disso, 
também são encontradas dificuldades perante a falta de capacitação dos profissionais para certas eventualidades, falta de material disponível nas unidades, e, sobretudo, a falta de organização das equipes diante da oferta dos cuidados (Pessalacia, Zoboli \& Ribeiro, 2016).

O presente estudo demonstrou que os pacientes em cuidados paliativos passam a maior parte do tempo em ambiente hospitalar, o que muitas vezes prejudica a assistência com base nos princípios paliativistas. No ano 2018 foi aprovada a Política Nacional de Cuidados Paliativos, que pactua uma meta para o SUS oferecer assistência ao paciente paliativo com qualidade em toda a Rede de Atenção à Saúde (Frossard, 2016), o que torna necessário melhorar a oferta de conhecimento sobre essa temática para as equipes da atenção primária. Isso pode ser feito através de programas nacionais vinculados às unidades básicas de saúde, que abranjam a formação e a capacitação profissional, a implementação de recursos para melhor assistir os pacientes, bem como o planejamento da assistência (Silva \& Dittz, 2018).

Diante disso, a equipe deve receber treinamentos e capacitações com foco em cuidado paliativo, mas voltando-o para as necessidades biopsicossoais afetadas, bem como o estímulo ao autocuidado e à qualidade de vida, evitando-se uma assistência fragmentada voltada para protocolos institucionais e que generalizam o cuidado ofertado.

Cabe ressaltar que no país os cuidados paliativos ainda não são regulamentados por lei, e os serviços que ofertam essa assistência são poucos, visto que ainda há um grande desconhecimento e preconceito relacionados a essa ótica de cuidado (ANCP, 2019). No entanto, existem normas que justificam e impõem a necessidade e a importância dessa prática descrita na Constituição Federal, Capítulo I, artigo $1^{\circ}$ e $5^{\circ}$ e no Código Civil Brasileiro, Capítulo II, artigo 15 (Brasil, 2020).

Ademais, o Código de Ética da Enfermagem dispõe em parágrafo único que o profissional de enfermagem tem o dever de respeitar as decisões do paciente e da família no que diz respeito ao seu tratamento, bem como o desejo de receber ou não procedimentos no momento que estiver incapacitado; também é dever da enfermagem, conforme artigo 39 e 40 , orientar paciente e família sobre os riscos e benefícios e sobre as possíveis consequências de procedimentos e de assistência, devendo respeitar a recusa do paciente e da família (Cofen, 2017).

Portanto, sabendo que apesar de toda sua história o cuidado paliativo teve sua ênfase nos últimos anos devido ao aumento da população idosa e à elevação do número de doenças crônicas não transmissíveis, torna-se necessária a ampliação do conhecimento acerca dessa temática por parte de todos os profissionais, principalmente da enfermagem, por ser a categoria que passa mais tempo à beira leito do paciente, prestando sua assistência e seus cuidados. Dessa forma, é visível a necessidade de novas condutas e novas técnicas, embasadas em políticas e resoluções sobre cuidados paliativos propriamente ditos (Leite et al., 2020).

\section{Conclusão}

Observou-se que os enfermeiros têm uma visão limitada do que são os cuidados paliativos, tendo em vista sua falta de treinamento ou capacitação, bem como a carência dessa temática durante a formação acadêmica, o que certamente indica que os enfermeiros não estão preparados para ofertar uma assistência de qualidade para pacientes paliativos, embora vivenciem-na diariamente.

Vale ressaltar que os participantes apontaram a importância de ter uma equipe multiprofissional e capacitada para esse tipo de cuidado, o que mostra o engajamento por parte deles para melhorar a assistência que ofertam. Observa-se a necessidade do desenvolvimento de pesquisas futuras em cuidados paliativos que incentivem uma reflexão quanto ao cuidado oferecido a estes pacientes, bem como o conhecimento dos profissionais sobre esta temática.

\section{Referências}

Academia Nacional de Cuidados Paliativos. ANCP e cuidados paliativos no Brasil. https://paliativo.org.br/cuidados-paliativos/cuidados-paliativos-no-brasil. 
Almeida, V. C., Gama, E. S. C., Espejo, C. A. N. \& Pedroso, J. S. (2018). A singularidade da dor de pacientes oncológicos em cuidados paliativos. Mudanças Psicologia da Saúde, 26 (1), 75-83.

Amorim, S. M., Mourão, C. C., Almeida, A. C. V., Leite, I. C. M., Oliveira, M. A. \& Faraco, R. L. P. S. (2020). Cuidados paliativos oncológicos na formação de enfermeiros: reflexões sob a ótica dos conceitos de instituição, instituído e instituinte da análise institucional. Research Society and Development, 9 (8), 124 .

Andrade, F. L. M., Silva, M. E. S., Macedo, E. L., Brito, D. T. F., Sousa, A. T. O., Agra, G. (2018). Dor oncológica: manejo clínico realizado por enfermeiros. Revista de Iniciação científica da Universidade Vale do Rio Verde, 8 (1), 1-13.

Aquino, A. T. T., Goés, I. M. C. \& Malcher, M. (2020). A percepção da equipe de enfermagem sobre cuidados paliativos e o manejo da dor na unidade de terapia intensiva do hospital Municipal de Santarém. Revista Enfermagem Brasil, 15 (6), 295-300.

Barbosa, R. P. S., Batista, J. B. V., Santos, B. M. P., Costa, M. I. A. C., Santos, M. S. L. \& Fernandes, M. A. (2020). Paciente com câncer na fase terminal de vida em cuidado paliativo: vivência do cuidador familiar. Revista online de pesquisa Cuidado é fundamental, 696-702.5.

Borba, J. C. Q. (2020). Pacientes sob cuidados paliativos em fase final de vida: vivencia de uma equipe multiprofissional. Trabalho de Conclusão de Curso, Especialização em Cuidados Paliativos, Universidade Federal de Paraíba, João Pessoa, PB, Brasil.

Brandão, M. L. A. \& Góis, R. M. O. (2020). Assistência de enfermagem para pacientes oncológicos em cuidados paliativos: importância da interação familiar no tratamento. Ciências Biológicas e da Saúde uni, 6 (1), 175-188.

Brasil (2020). Manual de Cuidados Paliativos. Hospital SírioLibanês; 1-171.

Carvalho, B. M., Vieira, R. M., Tacla, M. T. G. M., Misael, E. B. P. B. \& Barros, N. G. (2020). Percepção de familiares de crianças internadas em unidade pediátrica sobre cuidados paliativos. Brazilian Journal of Development, 6 (1), 74424-74438.

Coelho, C. B. T. \& Yankaskas, J. R. (2017). Novos conceitos em cuidados paliativos na unidade de terapia intensiva. Revista Brasileira de Terapia Intensiva, $29(2), 222-230$.

Conselho Federal de Enfermagem. (2017). Resolução Cofen $n^{\circ}$ 564/2017. http://www.cofen.gov.br/resolucao-cofen-no-5642017_59145.html.

Corgozinho, M. M., Barbosa, L. O., Araújo, I. P. \& Araújo, G. T, F. (2020). Dolor y sufrimento desde la perspectiva de la atencion centrada en el paciente. Revista Bioética, 28 (2), 249-256.

Frossard, A. (2016). Os cuidados paliativos como política pública: notas introdutórias. Cad. EBAPE.BR,14, 640-655

Gomes, A. Z. \& Othero, M. B. (2016). Cuidados Paliativos. Estudos Avançados, 30 (88), 155-166.

Gomes, C. N. (2019). Cuidado paliativo em idoso no ambiente domiciliar. Trabalho de Conclusão de Curso, SI, Itupivara, Brasil.

Hall, S., Petkova, H., Tsouros, A. D., Constantini, M. \& Higgson, I. J. (2011). Paliative care or older people: better practices. Estados Unidos: Word Health Organization.

Leite, A. C., Pilar, T. M, I. V., Prudêncio, L. D., Almeida, D. S., Mendes, A. M., Sousa, B. B., Barbosa, M. V. R. \& Vianas, M. R. P. (2020). Atribuições do enfermeiro nos cuidados paliativos em oncologia pediatra. Brazilian Journal of Development. 5 (10), $79459-79474$.

Lima, O. Q. C. (2019). Comunicação promovida por uma equipe multidisciplinar ao paciente com câncer em cuidados paliativos. Trabalho de Conclusão de Curso, Universidade Federal da Paraíba. João Pessoa, PB, Brasil.

Lóos, J. C. S., Dias, V. E., Cabral, H. L. T. B. \& Souza, C. H. M. (2020). Os saberes da saúde e a interdisciplinaridade nos cuidados paliativos. Interdisciplinary Scientific Journal, 5 (5), 250.

Lourençato, F. M. (2020). Implantação de um serviço de cuidados paliativos no serviço de urgência e emergência de um hospital público universitário. São Paulo, 1-114.

Malta, D. C., Bernal, R. T. I., Neto, E. V., Curci, K. A., Pasinato, M. T. M., Lisboa, R. M., Cachapuz, R. F., Coelho, K. S. C., Santos, F. P. \& Freitas, M. I. F. (2020). Doenças crônicas não transmissíveis e fatores de risco e proteção em adultos com ou sem plano de saúde. Ciência \& Saúde Coletiva. 25 (8), 2973 2983.

Martins, J. C., Silva, B. R., Strieder, C. P., Silveira, I. I., Camilo, K. C. \& Nunes, T. C. (2020). Família e câncer: considerações acerca do diagnóstico, tratamento e terminalidade. Salão do Conhecimento, Unijuc, 1-6.

Meireles, D. S., Bittencourt, G. R. G. D., Montenegro, C. P. D., Fortunato, C. N. \& Melo, G. M. O. G. (2020). Assistência de enfermagem ao idoso em cuidados paliativos: um relato de experiência. Brazilian Journal of Development, 6 (6), 40854-40867.

Miranda, G. M. D., Mendes, A. C. G. \& Silva, A. L. A. (2016). O envelhecimento populacional brasileiro: desafios e consequências sociais atuais e futuras. Revista Brasileira de geriatria e gerontologia. 13 (3), 507-519.

Monteiro, F. L. R., Queiroz, J. C., Couto, A. C. A., Oliveira, L. G., Pinto, B. F., Fagundes, L. C. M., Pinheiro, F. P. \& Gama, R. L. (2020). Atuação da equipe multiprofissional em cuidados paliativos oncológicos na assistência domiciliar ao paciente e seus familiares. Brazilian Journal of Development, 6 (5), 31203169.

Nascimento, I. C. C., Campos, J. S., Vieira, V. P. \& Barbosa, M. C. R. (2020). Percepção da enfermagem sobre avaliação da dor oncológica. Revista de ciências biológicas e da saúde. 32 (10), 51-61. 
Nascimento, J. C. C. (2017). Avaliação da dor em paciente com câncer em cuidado paliativo, a luz da literatura. Revista Acadêmica do Instituto de Ciências da Saúde, 3 (1). 1-16.

Oliveira, N. J. J., Oliveira, S. B. S., Migowsk, E. R. \& Riegel, F. (2017). O papel da enfermagem no tratamento não farmacológico da dor de pacientes oncológicos. Revista Dor, 18 (3), 261-5.

Paiva, C. F., Santos, T. C. F., Montenegro, H. R. A., Costa, R. \& Almeida, A. J. F. (2019). Reconfiguração dos cuidados paliativos de enfermagem oncológica: contribuições da enfermagem. Revista Brasileira de Enfermagem, 73 (6), 1-8.

Pereira, A. S., Shitsuka, D. M., Parreira, F. J., Shitsuka R. (2018). Metodologia da Pesquisa Científica. Santa Maria: UFSM, NTE.

Pessalacia, J. D. R., Zoboli, E. L. C. P. \& Ribeiro, I. K. (2016). Equidade no acesso aos cuidados paliativos na atenção à saúde: uma reflexão teórica. Revista de Enfermagem do Centro-Oeste Mineiro, 1 (6), 2119-2139.

Pires, I. B., Menezes, T. M. O., Cerqueira, B. B., Albuquerque, R. S., Moura, H. C. G. B., Freitas, R. A., Santos, A. L. S. \& Oliveira, E.S. (2019). Conforto no final de vida na terapia intensiva: percepção da equipe multiprofissional. Acta Paulista Enfermagem, 3, 1-17.

Pulga, G., Cassol, L., Amaral, M., Januário, A. G. F., Feldkerchen, N. \& Nodori, T. M. S. (2019). O trabalho da equipe multidisciplinar na melhoria da qualidade de vida de pacientes em estágio terminal com foco nos cuidados paliativos. Unoesc \& Ciência, 5 (2), 163 -168.

Pupim, C. T. (2018). Cuidados Paliativos em geriatria: concepção da equipe multiprofissional. Programa de Pós-Graduação. Vitória, 1-62.

Queiroga, N. M., Menezes, L. V., Lima, J. M. R. \& Andrade, D. D. B. C. (2020). Cuidados paliativos de idosos no contexto da atenção primária à saúde: uma revisão de literatura. Brazilian Journal of Development, 6 (6), 38821-38832.

Queiroz, T. A., Ribeiro, A. C. M., Guedes, M. V. C., Coutinho, D. T. R., Galiza, F. T. \& Freitas, M. C. (2018). Cuidados Paliativos ao idoso na terapia intensiva: olhar da equipe de enfermagem. Texto \& Contexto Enfermagem, 27 (1), 1-10.

Ribeiro, A. S. P. (2017). Prática de cuidados paliativos em idosos com demência. Trabalho de Conclusão de Curso, Universidade Brasileira, 1-20.

Santo, L. F. S. E., Ferreira, J. I. C., Pacheco, Q. C. \& Souza, S. R. (2020). Os desafios dos enfermeiros de cuidados paliativos no cenário hospitalar: revisão integrativa. Revista Eletrônica Acervo e Saúde, Susp (49), 1-9.

Santos, A. L. N., Lira, S. S. \& Coata, R. S. L. (2018). Cuidados Paliativos prestados pelo enfermeiro ao paciente oncológico. Revista Dê Ciência em foco, 2 (1), 63-77.

Santos, B. C., Souza, M., Scaldelai, R. S., Lozano, T. S. P., Sailer, C. \& Preto, V. A. (2017.) A percepção dos enfermeiros de um hospital geral sobre os cuidados paliativos. Revista de Enfermagem UFPE, Recife, 11 (6), 2288-5.

Santos, F. L. E. P., Silva, M. T. \& Ribeiro, A. S. (2020). A percepção biopsicosocioespiritual do processo de finitude na tríade enfermeiro x paciente x família. Research Society and Develeopment, 9 (8) 1-6.

Santos, J. P. R., Pedroso, M. D., Carvalho, A. C. M., Farias, C. B., Freitas, E. A. C., Cordeiro, J. M. G. \& Dias, L. G. S. (2020). Cuidados paliativos em neonatologia: uma revisão narrativa. Brazilian Journal of health review, 3 (5), 14589-14601.

Silva, A. E., Braga, P. P., Sena, R. R., Duarte, E. D. \& Sena, L. R. (2020). Cuidados paliativos domiciliares: revisão integrativa. Ciência Cuidado e Saúde, 1 (8), 1-7.

Silva, A. E. \& Dittz, E. D. (2018). Cuidado Paliativo na atenção domiciliar: conhecimento, definição e elegibilidade na perspectiva do profissional de saúde. Anais do Colóquio Panamericano de Investigación in Enfermaría, Cuba, 16.

Silva, C. V. \& Gaspadini, I. B. (2020). A influência da participação familiar no tratamento do paciente oncológico. Revista Ciência e humanização, 1 (1), 7488 .

Silva, D. N. O., Paz, L. S., Vieira, S. S., Ferreira, A. M. V. \& Neves, W. A. J. (2020). A enfermagem oncológica nos cuidados paliativos: uma revisão sistemática integrativa. Revista Portal Saúde e Sociedade, 5 (1), 1363-1379.

Silva, F. C. F. S., Cunha, C. S. Feitosa, T. S. R. G. T., Silva, A. D. M. \& Sousa, I. S. B., (2020). Assistência de enfermagem a pacientes com câncer em cuidados paliativos: revisão integrativa. Revista Enfermagem Atual, 9 (21) 149-157.

Silveira, N. R., Nascimento, E. R. P., Rosa, L. M., Jung, W., Martins, S. R. \& Fontes, M. S. (2016). Cuidado paliativo e enfermeiros da terapia intensiva: sentimentos que ficam. Revista Brasileira de Enfermagem, 69 (6), 1074-81.

Silveira, P. J. da, Costa, A. E. K. da, Lohmann, P. M., \& Lavall, E. (2020). Revisão integrativa: cuidados paliativos em pacientes oncológicos. Research, Society and Development, 9(2), e144922136.

Simão, V. M., Mioto, R. C. (2016). O cuidado paliativo e domiciliar em países da América Latina. Saúde Debate, 40 (8),156-169.

Sousa, A. D. P. \& Nunes, N. A. H. (2019). Assistência do profissional enfermeiro no manejo da dor de pacientes sem possibilidades terapêuticas de cura. Revista de Enfermagem UFPI, 8 (4), 96-101. 